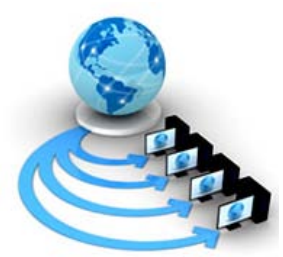

Volume 8, No. 7, July - August 2017

International Journal of Advanced Research in Computer Science

RESEARCH PAPER

\author{
Available Online at www.ijarcs.info
}

\title{
IRIS RECOGNITION USING MODULAR NEURAL NETWORK AND FUZZY INFERENCE SYSTEM BASED SCORE LEVEL FUSION
}

\author{
Arif Iqbal Mozumder and Shahin Ara Begum \\ Department of Computer Science, Assam University, Silchar, India
}

\begin{abstract}
Iris Recognition System (IRS) is one of the prominent recognition system used in various application domains such as border control, access control, airports and citizen registration to accurately identify a person. The IRS generally consists of four steps viz. Image Acquisition, Feature Extraction, Segmentation and Recognition. This paper proposes an approach for recognition of an iris using Modular Neural Network and Fuzzy inference system based Score Level Fusion. The performance of the proposed approach is tested on the standard iris database, IITD, obtained from the public domain digital repository. The experimental results demonstrate the efficiency of the proposed approach for identification and verification of an iris image with different performance measures over the considered dataset.
\end{abstract}

\section{Keywords: Iris recognition, Artificial Neural Network, Fuzzy Inference System, Discrete Cosine Transform, Score Level Fusion}

\section{INTRODUCTION}

To deal with the secure and precise recognition of a person in today's technological world, the recognition system based on physiological and behavioral characteristics are used in different application domains such as health care, citizen registration, passport, border control, access control etc. [1]. Banking sector is also initiating to install the IRS in the ATMs to replace the traditional PIN based verification during transaction [2]. Numerous biometric systems based on different biometric characteristics such as face, iris, palm, finger-print, retina, voice and gait are proposed by various authors till today. Among all the biometric systems, the research reveals that the system based on iris is more reliable and secure due to its non-invasiveness and consistencies. Iris, an externally visible internal organ of the body comprising of unique complex structures, does not require any contact with the capturing device during image acquisition and its complex structure remain same throughout one's life span after the age of one [3]. The Iris's complex structure provides unique texture information different for each eye even among the eyes of same person [4].

Typically, Iris recognition system consists of four steps viz. Image Acquisition, Segmentation, Feature Extraction and Recognition as shown in Fig. 1. Each of these steps play a pivotal role in the recognition system. The system has two modules viz. enrolment and recognition module. During enrollment, iris image features are extracted and stored in the database as a reference. During recognition, features from query iris is extracted and compared with features stored in the database $[5,6]$.

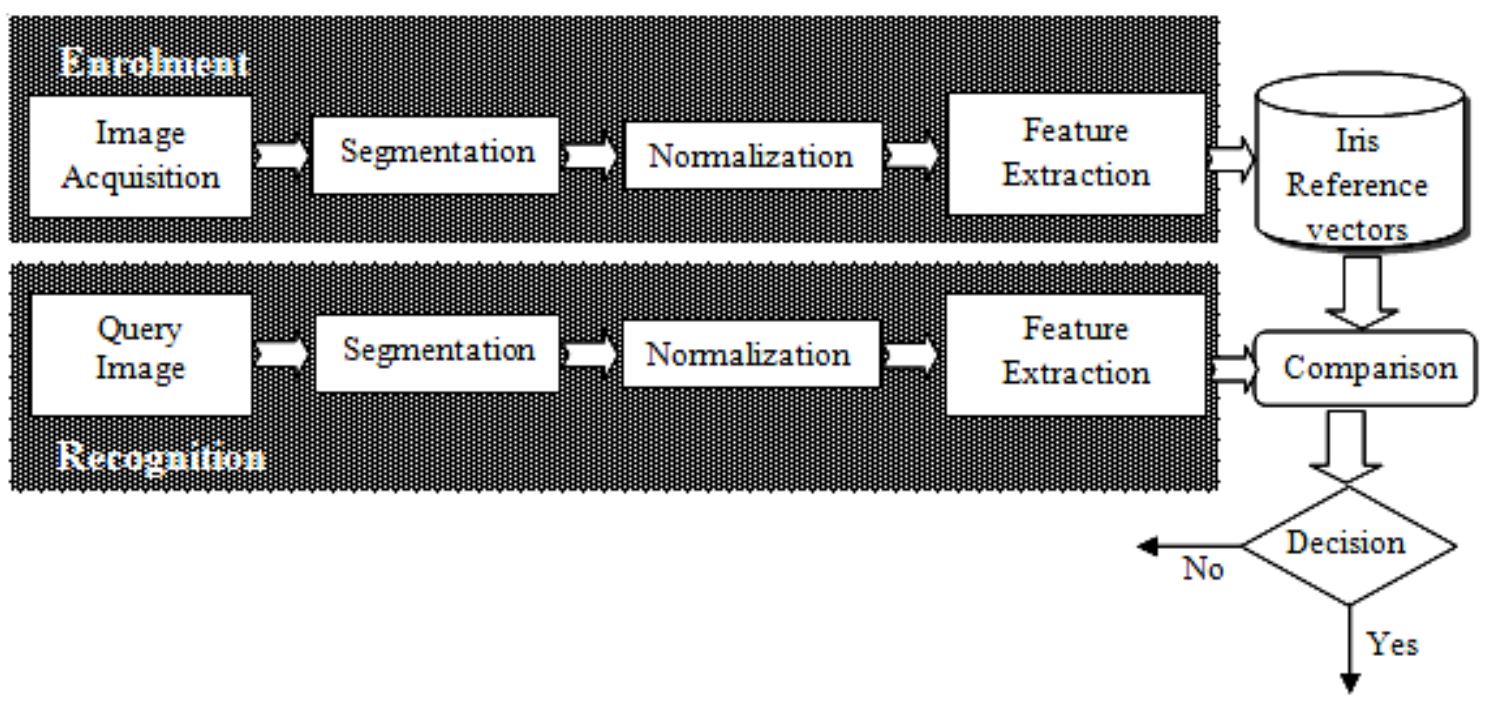

Fig. 1 Iris Recognition System

The task of IRS is to recognize an iris precisely and accurately with less False Accept Rate (FAR) and False Rejection Rate (FRR). The iris, annular part between sclera and pupil, is segmented from rest of the eye image which may contain noises such as eyelashes, eyelids, reflections etc. These noises damage the texture information which in 
turn increases the FAR and FRR of the system. To deal with this, the idea of fusion strategy has been adopted by different authors. Rahulkar et al. [7], proposed score level fusion strategy using $k$-out-of- $n$ : $A$, a post classifier, to reduce the FRR of the recognition system. Park et al. [8] used Hamming Distance and support vector machine to perform score level fusion. The authors Shin et al.[9], Santos et al. [10] presented an iris recognition system based on score level fusion strategy in an unconstraint environment. Marsico [11] presented iris matching approach based on combination of LBP and discriminable textons (BLOBs), local features of an iris. The fusion strategy is also adopted by Eskandari et al. [12], Rabiul Islam [13], Ganorkar et al. [14], Thul et al. [15] and Madane et al. [16], Mozumder et al. [17] for accurate recognition of an iris image.

The present research work deals with recognition of an iris where score level fusion strategy based on fuzzy inference system has been adopted in order to reduce the FAR and FRR of the system. Modular Neural Networks have been used as a recognizer whose outputs are fused together with the fuzzy inference system to determine the actual class (identity) of an iris. The system consists of six modules of neural networks and the fuzzy inference system. During enrolment, iris image is divided into six segments and each Module is trained with their corresponding segment. During recognition, six segments of query iris are fed into their corresponding trained module where each module responds with the class of the segments. These outputs go through the fuzzy inference process for final recognition. The rest of the paper is organized as follows: Section 2 describes Segmentation and Normalization followed by Feature Extraction and Recognition in section 3 and section 4 . Section 5 describes the experimental result followed by conclusion in section 6 .

\section{SEGMENTATION AND NORMALIZATION}

Segmentation means the isolation of an iris part from the rest of the eye image. It is one of the vital steps for iris recognition system which may affect the overall performance of the system. The more accurate the segmentation better will be the recognition performance. Due to inconsistencies of size of the iris, normalization is done to make the iris size uniform for better recognition accuracy.

This paper mainly focuses on the recognition step of the system. The segmentation and normalization is done according to the previous work based on Quantum-behaved
Particle Swarm Optimization (QPSO) explained in [18]. The sample of segmented iris is shown in fig. 2 .
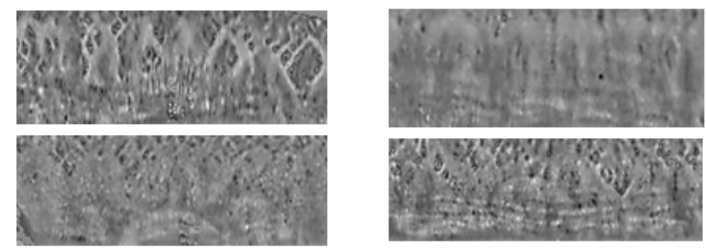

Fig. 2 Sample of segmented and normalized iris from IITD database

\section{FEATURE EXTRACTION}

For a set of $N$ classes with $n$ iris images per class, DCT features are extracted from each of the images according to the algorithm described in [17].

Let

$$
F_{i}=\left[f_{i 1}, \ldots, f_{i j}, \ldots, f_{i n}\right]
$$

be an $N \times n$ DCT feature matrix of $i^{\text {th }}$ class where, $f_{i j}$ denotes the features of $j^{t h}$ iris of $i^{t h}$ class. The reference vector, $F$, of all training iris images are defined as the concatenation of $F_{i}, i=1,2, \ldots, n$ as

$$
\begin{gathered}
F=\left[F_{1}, F_{2}, \ldots, F_{N}\right] \\
=\left[f_{11}, \ldots, f_{i j}, \ldots, f_{i n}\left|f_{21}, \ldots, f_{2 j}, \ldots, f_{2 n}\right| \ldots \mid f_{N 1}, \ldots, f_{N j}, \ldots, f_{N}\right.
\end{gathered}
$$

This reference vector, F, is used to train the modular neural networks.

\section{RECOGNITION}

In the present work, fuzzy inference system is incorporated into the output of modular neural network for recognition of an iris image. The proposed recognition approach is shown in Fig. 3. Each iris image is divided into six segments. The DCT features are extracted from each segment and reference vector, $F$, is constructed as described in section 3 . The system consists of six modules and the fuzzy inference system. Each ANN module is trained with their corresponding segment features. More details about training can be found in [17].

During recognition, DCT features of each segment of a query iris are presented into the corresponding trained modules for recognition. Each module responds with the class, $y_{i}$, of the segment. These outputs are fed into the fuzzy inference system along with the target vectors. 


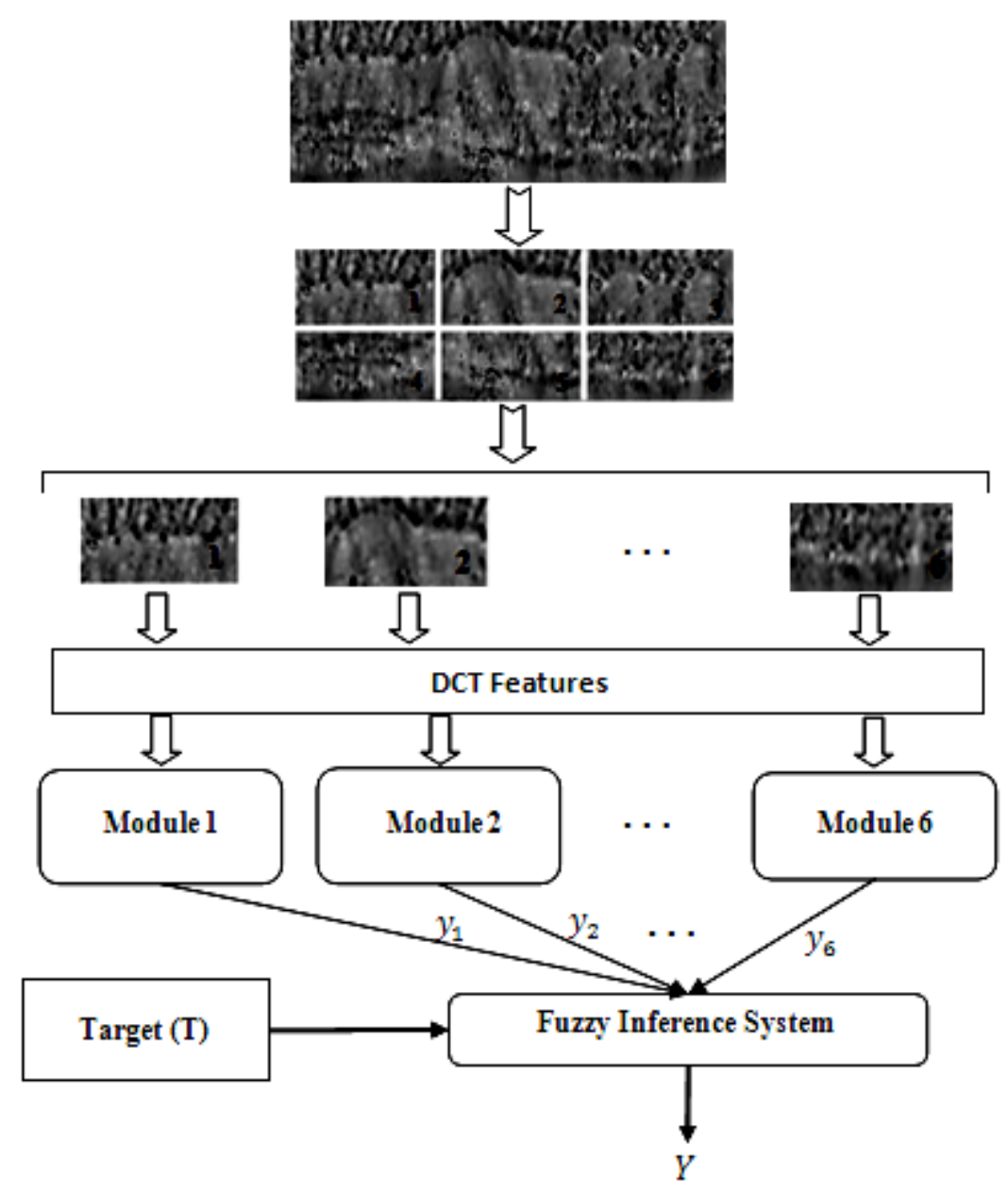

Fig. 3 Proposed recognition approach

Firstly, Fuzzy inference system, defines a fuzzy set, $Y$, in $T$ on the output of each module $y$ as [19]:

$$
Y=\left\{\left(y, \mu_{Y}(y)\right) \mid y \in T\right\}
$$

where, $T$ is the universal set of vectors representing the classes of iris stored as a reference and $\mu_{Y}(y)$ is the membership value of $Y$ defined by:

$$
\mu_{Y}(y)=\left\{\begin{array}{lr}
0, & 0 \leq s \leq a \\
2 \times\left(\frac{s-a}{1-a}\right)^{2}, & a \leq s \leq \frac{a+1}{2} \\
1-2 \times\left(\frac{s-1}{1-a}\right)^{2}, & \frac{a+1}{2}<s \leq 1
\end{array}\right.
$$

where, $a$ denotes threshold value and $s$ denotes the similarity rate calculated by:

$$
s=1-\sqrt{(y-t)^{2}}
$$

where, $t$ represents the target. Next, the inference system chooses the output of modules having membership value, $\mu_{Y}$, greater than zero and discards the rest. Finally, voting operation is performed among the selected output for final recognition of an iris.

\begin{tabular}{|c|c|c|c|c|c|}
\hline Query Image & Segments & $\begin{array}{c}\text { Output } \\
(y)\end{array}$ & $\begin{array}{l}\text { Membership } \\
\text { value, } \mu_{y}\end{array}$ & $\begin{array}{c}\text { Selected } \\
\text { Output }\end{array}$ & $\begin{array}{l}\text { Recognized } \\
\text { class }\end{array}$ \\
\hline & & 1 & 0.25 & $\sqrt{ }$ & 1 \\
\hline \multirow[t]{3}{*}{ (class 1) } & & 1 & 0.71 & $\sqrt{ }$ & \\
\hline & & 1 & 0.41 & $\sqrt{ }$ & \\
\hline & & 1 & 0 & $x$ & \\
\hline
\end{tabular}
The example of recognition procedure is shown in Table. 1.

Table 1 Example of iris recognition procedure 


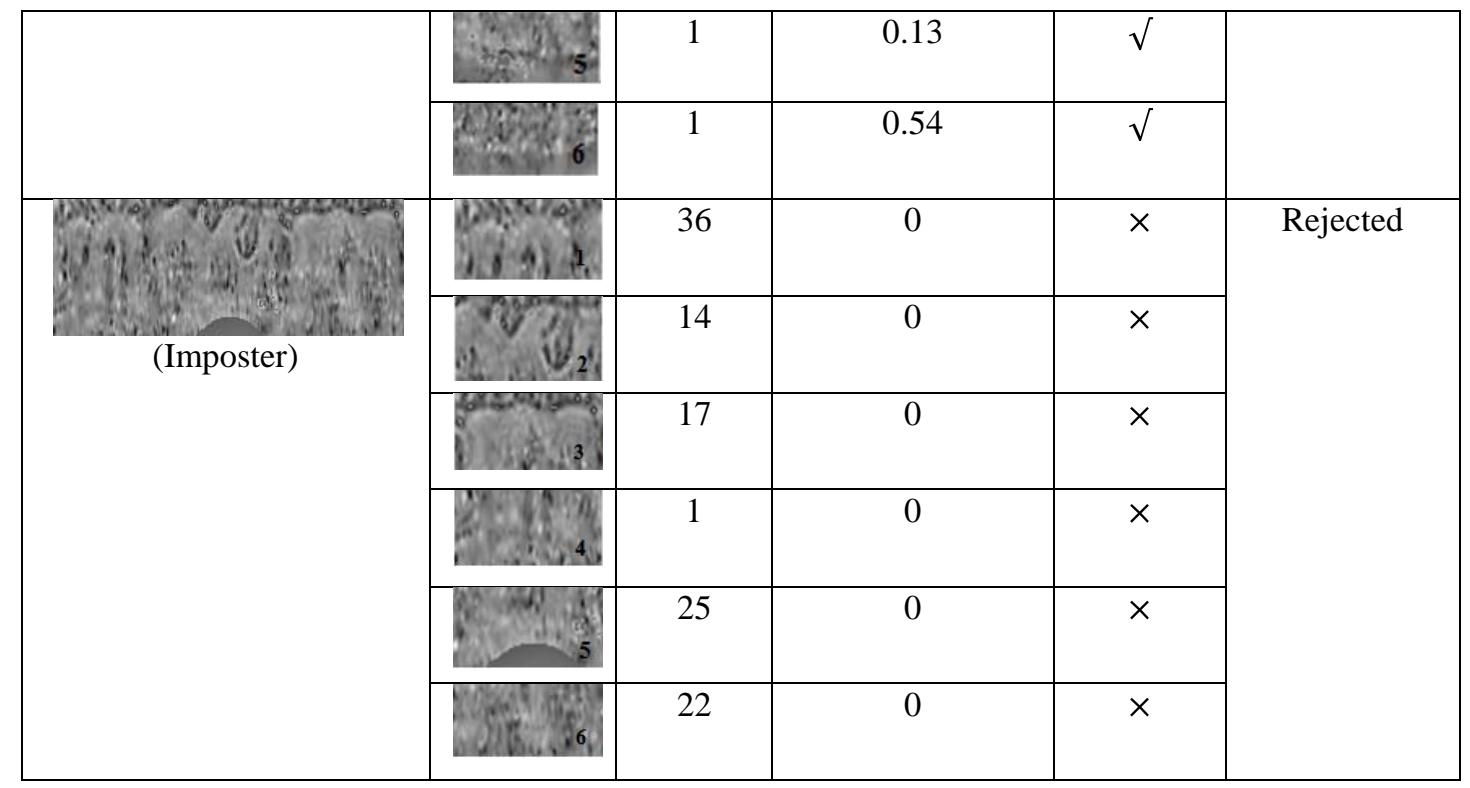

With incorporation of fuzzy inference system, any noisy or misclassified segment can be discarded, before the score fusion, using membership value $\mu_{Y}$ as because these segments most probably will have the membership value zero. As a result of which recognition performance of the system improves considerably. This approach is also capable of rejecting the imposters more accurately as is revealed in the experimental result.

\section{EXPERIMENTAL RESULTS}

The experiments were performed on Windows 7 environment with Intel i3 processor $(2.4 \mathrm{GHz})$ and $3 \mathrm{~GB}$ RAM using MATLAB 7.0 software. The proposed recognition approach was evaluated on IITD [19]. The left eye of each person is selected for experimentation and the division of the IITD database for a different purpose during experiments is tabulated in table 2 .

Table 2 Division of IITD iris database

\begin{tabular}{|l|c|c|c|}
\hline Purpose & No. of Sample & No. of image/sample & Total \\
\hline Training & 90 & 4 & 360 \\
\hline Testing & 90 & 1 & 90 \\
\hline Imposter & 50 & 3 & 150 \\
\hline
\end{tabular}

The architecture of the module used in the present work consists of five small Multi-Layer Perceptron (MLP) neural networks. Each MLP consists of input, hidden and output layer with 50, 24 and 1 neurons respectively. The optimal parameters of MLP are tabulated in table 3. The average training and validation performance of the MLPs are presented in Fig. 4 and table 4.

Table 3 optimal parameters of MLP

\begin{tabular}{|l|l|}
\hline Training Function & 'trainrp' \\
\hline Transfer function & $\begin{array}{l}\text { 'tansig' for first layer } \\
\text { 'purelin' for second layer }\end{array}$ \\
\hline Learning rate & 0.30 \\
\hline Epochs & 2000 \\
\hline Error Goal & 0.0001 \\
\hline Minimum gradient & 0.0001 \\
\hline
\end{tabular}

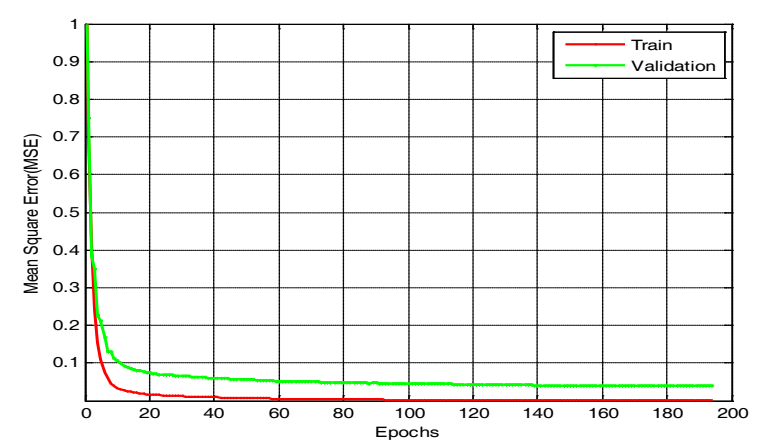

Fig. 4: Training and Validation performance.

Table 4. Network training performance

\begin{tabular}{|c|c|c|c|}
\hline $\begin{array}{c}\text { No. of } \\
\text { Hidden } \\
\text { neuron }\end{array}$ & MAE & RMSE & Accuracy \\
\hline 21 & 0.0535 & 0.0085 & 88.68 \\
\hline 22 & 0.0635 & 0.0087 & 89.73 \\
\hline 23 & 0.0455 & 0.0086 & 98.68 \\
\hline $\mathbf{2 4}$ & $\mathbf{0 . 0 5 8 0}$ & $\mathbf{0 . 0 0 5 5}$ & $\mathbf{1 0 0}$ \\
\hline 25 & 0.1205 & 0.03267 & 100 \\
\hline 26 & 0.0657 & 0.0122 & 94.73 \\
\hline 27 & 0.0552 & 0.0108 & 98.68 \\
\hline 28 & 0.0756 & 0.0139 & 88.15 \\
\hline 29 & 0.0364 & 0.0055 & 95.73 \\
\hline 30 & 0.0643 & 0.01396 & 97.36 \\
\hline
\end{tabular}

The recognition of an iris is done with the help of trained modules and fuzzy inference system as explained in section 4. The FAR, FRR, Recognition Accuracy and execution time are used as performance measures in the proposed recognition approach. The threshold value, $a$, for class separation is determined from FAR and FRR of the system. To evaluate the proposed recognition approach, its performance is compared with the existing recognition approaches viz. Masek [20], Rai et al. [21], and Pirale et al. 
[22]. The recognition performances is evaluated in both verification and identification mode. The False Accept Rate (FAR), False Reject Rate (FRR) and accuracy are calculated as [23]

$$
F A R=\frac{\text { No. of times imposter accepted }}{\text { Total no. of comparison }}
$$

$$
\begin{gathered}
F R R=\frac{\text { No. of times pretender rejected }}{\text { Total no. of comparison }} \\
\text { Accuracy }=\left(1-\frac{F A R+F R R}{2}\right) \times 100
\end{gathered}
$$

The Fig. 5 represents the FAR and FRR at different threshold value, $a$.

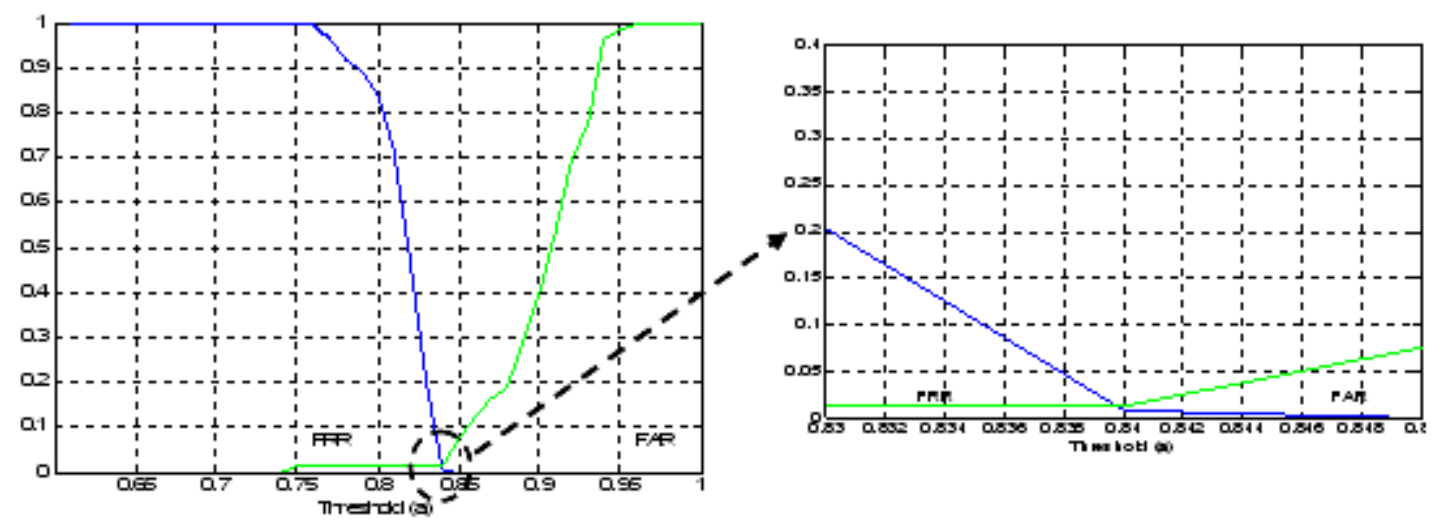

Fig. 5 FAR and FRR at different threshold value, $a$

It is concluded from Fig. 5 that the value, $a$, equal to 0.84 is suitable to be choosen as a threshold value for class separation.

\subsection{Verification performance}

During Verification, system tries to match query iris with specific iris template from the database (one-to-one comparison). The proposed approach achieves $99.06 \%$ of verification accuracy on the considered dataset. The recognition performance on verification mode with different value of ' $a$ ' is presented in table 5.

Table 5 Recognition performance on verification mode

\begin{tabular}{|c|c|c|c|}
\hline Threshold (a) & FAR & FRR & Accuracy (\%) \\
\hline 0.81 & 0.1463 & 0.0105 & 92.1566 \\
\hline 0.82 & 0.0894 & 0.0105 & 95.0021 \\
\hline 0.83 & 0.0243 & 0.0105 & 98.2541 \\
\hline $\mathbf{0 . 8 4}$ & $\mathbf{0 . 0 0 8 1}$ & $\mathbf{0 . 0 1 0 5}$ & $\mathbf{9 9 . 0 6 7 1}$ \\
\hline 0.85 & 0 & 0.0526 & 97.3684 \\
\hline 0.86 & 0 & 0.1052 & 94.7368 \\
\hline 0.87 & 0 & 0.1368 & 93.1578 \\
\hline 0.88 & 0 & 0.1578 & 92.1052 \\
\hline 0.89 & 0 & 0.2315 & 88.4210 \\
\hline 0.9 & 0 & 0.3263 & 83.6842 \\
\hline
\end{tabular}

\subsection{Identification performance}

During Identification, the system tries to find query iris from entire iris templates stored in the database as references (one-to-many comparison). The proposed approach achieves the identification accuracy of $98.96 \%$ with FAR=0.0081 on the considered dataset. The recognition performance on identification mode of the proposed approach with different values of ' $a$ ' is presented in table 6 .

Table 6 Recognition performance on identification mode

\begin{tabular}{|c|c|c|c|}
\hline Threshold (a) & FAR & FRR & Accuracy (\%) \\
\hline 0.81 & 0.7235 & 0.0125 & 63.19 \\
\hline 0.82 & 0.4796 & 0.0125 & 75.39 \\
\hline 0.83 & 0.2032 & 0.0125 & 89.21 \\
\hline $\mathbf{0 . 8 4}$ & $\mathbf{0 . 0 0 8 1}$ & $\mathbf{0 . 0 1 2 5}$ & $\mathbf{9 8 . 9 6}$ \\
\hline 0.85 & 0.0000 & 0.0750 & 96.25 \\
\hline 0.86 & 0.0000 & 0.1250 & 93.75 \\
\hline 0.87 & 0.0000 & 0.1625 & 91.87 \\
\hline 0.88 & 0.0000 & 0.1875 & 90.62 \\
\hline 0.89 & 0.0000 & 0.2875 & 85.62 \\
\hline 0.9 & 0.0000 & 0.4000 & 80.00 \\
\hline
\end{tabular}




\subsection{Comparative study}

In order to evaluate the proposed recognition approach, the performance of the proposed approach is compared with the performances of the existing approaches viz. Masek[19], Rai et al. [20] and Pirale et al. [21]. The comparison result with respect to recognition accuracy during verification and identification and execution time is presented in table 7 .

Table 7 The comparison of result with existing methods

\begin{tabular}{|l|c|c|c|c|}
\hline \multirow{2}{*}{ Method } & \multicolumn{2}{|c|}{ Recognition Accuracy } & \multicolumn{2}{c|}{ Execution time (approx.) } \\
\cline { 2 - 5 } & $\begin{array}{c}\text { Verification } \\
\mathbf{( \% )}\end{array}$ & $\begin{array}{c}\text { Identification } \\
\mathbf{( \% )}\end{array}$ & $\begin{array}{c}\text { Verification } \\
\text { (Sec.) }\end{array}$ & $\begin{array}{c}\text { Identification } \\
\text { (Sec.) }\end{array}$ \\
\hline Masek [19] & 98.67 & 98.10 & 0.11 & 13.80 \\
\hline Rai et al. [20] & 95.85 & 92.68 & 0.13 & 11.20 \\
\hline Pirale et al. [21] & 93.57 & 91.57 & 2.45 & 2.89 \\
\hline $\begin{array}{l}\text { Proposed Recognition } \\
\text { Approach }\end{array}$ & $\mathbf{9 9 . 0 6}$ & $\mathbf{9 8 . 9 6}$ & $\mathbf{0 . 6 9}$ & $\mathbf{2 . 4 6}$ \\
\hline
\end{tabular}

From table 7, it is concluded that the proposed approach outperform the existing approaches in terms of verification and identification accuracies and execution time on the considered dataset with respect to overall performances.

\section{CONCLUSION}

In the present work, fuzzy inference system is used as a fusion strategy during iris recognition. A set of modular neural networks are used as a classifier whose outputs are processed by fuzzy inference system for accurate recognition of an iris. The standard IITD iris database has been used to evaluate the proposed recognition approach. The proposed approach achieved $99.06 \%$ and $98.86 \%$ during verification and identification respectively with the considered dataset. This approach also outperforms the existing approaches proposed by Masek [19], Rai et al. [20] and Pirale et al. [21] in terms of recognition accuracy and execution time. The experimental results demonstrate the efficiency of the proposed iris recognition approach. In order to achieve the high performance robust iris recognition system, future work will be to incorporate Deep Learning Neural Network into the system for iris feature extraction and analyze the performance on both identification and verification mode.

\section{ACKNOWLEDGEMENT}

One of the authors (A. I. Mozumder) gratefully acknowledges UGC for the award of Maulana Azad National Fellowship for the research work.

\section{REFERENCES}

[1]. Farmanullah Jan, Segmentation and localization schemes for non-ideal iris biometric systems, Signal Processing, http://dx.doi.org/10.1016/j.sigpro.2016.11.007.

[2]. Lee J., "More Banks Turn to Biometrics to Keep an Eye on Security", Nerdwallet Banking. 20th May, 2017. https://www.nerdwallet.com/blog/banking/biometricswhen-your-bank-scans-your-voice-face-or-eyes.

[3]. Jhamb, M. and Khera, V.K., 2011. Iris based human recognition system. International Journal of Biometrics and Bioinformatics (IJBB), 5(1), p.1.

[4]. Monro, D.M., Rakshit, S. and Zhang, D., 2007. DCT-based iris recognition. IEEE Transactions on Pattern Analysis and Machine Intelligence, 29(4).

[5]. Daugman, John. "How iris recognition works." IEEE Transactions on circuits and systems for video technology 14, no. 1 (2004): 21-30.
[6]. Boles, Wageeh W., and Boualem Boashash. "A human identification technique using images of the iris and wavelet transform." IEEE transactions on signal processing 46, no. 4 (1998): 1185-1188.

[7]. Rahulkar, A.D., Jadhav, D.V. and Holambe, R.S., 2012. Fast discrete curvelet transform based anisotropic iris coding and recognition using k-out-of-n: A fused postclassifier. Machine Vision and Applications, 23(6), pp.1115-1127.

[8]. Park, H.A. and Park, K.R., 2007. Iris recognition based on score level fusion by using SVM. Pattern Recognition Letters, 28(15), pp.2019-2028.

[9]. Shin, K.Y., Nam, G.P., Jeong, D.S., Cho, D.H., Kang, B.J., Park, K.R. and Kim, J., 2012. New iris recognition method for noisy iris images. Pattern Recognition Letters, 33(8), pp.991-999.

[10]. Santos, G. and Hoyle, E., 2012. A fusion approach to unconstrained iris recognition. Pattern Recognition Letters, 33(8), pp.984-990.

[11]. De Marsico, M., Nappi, M. and Riccio, D., 2012. Noisy iris recognition integrated scheme. Pattern Recognition Letters, 33(8), pp.1006-1011.

[12]. Eskandari, M. and Toygar, Ö., 2013. Score Level Fusion for Face-Iris Multimodal Biometric System. In Information Sciences and Systems 2013(pp. 199-208). Springer International Publishing.

[13]. Islam, M., 2014. Feature and score fusion based multiple classifier selection for iris recognition. Computational intelligence and neuroscience, 2014, p.10.

[14]. Ganorkar, S.R. and Ligade, A.P., Enhancing the Performance of Iris Recognition System using Matching Score Fusion Technique.

[15]. Thul, S.V., Rishishwar, A. and Trivedi, M., Performance of Identification System Based on Score Level Fusion.

[16]. Madane, M. and Thepade, S., 2016. Score Level Fusion Based Bimodal Biometric Identification Using Thepade's Sorted n-ary Block Truncation Coding with Variod Proportions of Iris and Palmprint Traits. Procedia Computer Science, 79, pp.466-473.

[17]. Mozumder, A.I. and Begum, S.A., 2016, December. An efficient approach towards iris recognition with modular neural network match score fusion. In Computational Intelligence and Computing Research (ICCIC), 2016 IEEE International Conference on (pp. 1-6). IEEE.

[18]. Mozumder, A. I, Begum, S., A., “An Iris Recognition System in the Framework of Quantum Behaved Particle Swan Optimization based Iris Segmentation”, unpublished.

[19]. Zimmermann, H.,J., 1996, Fuzzy Set Theory and Its Applications, Thrid Ed., Springer, pp.11-21.

[20]. IITD Iris Database, http://web.iitd.ac.in/ biometrics/Database_Iris.htm, 2008

[21]. Masek, L. and Kovesi, P., 2003. Matlab source code for a biometric identification system based on iris patterns. The 
School of Computer Science and Software Engineering, The University of Western Australia, 2(4).

[22]. Rai, H. and Yadav, A., 2014. Iris recognition using combined support vector machine and Hamming distance approach. Expert systems with applications, 41(2), pp.588593.
[23]. Pirale, D., Nirgude, M., Gengje, S., 2016. Iris Recognition using Wavelet Transform and Neural Networks. International Journal of Science and Research (IJSR). 5(5), pp. 1055-1060.

[24]. Bodade, R.M. and Talbar, S.N., 2014. Iris Analysis for Biometric Recognition Systems. Springer. 